\title{
A Cross-Sectional Study on Cognitive Errors and Obsessive-Compulsive Disorders among Young People During the Outbreak of Coronavirus Disease 2019
}

\author{
Elham Darvishi ${ }^{1} \cdot$ Solmaz Golestan ${ }^{2} \cdot$ Farangis Demehri $^{3} \cdot$ Sheida Jamalnia $^{4}$ \\ Received: 13 July 2020 / Revised: 6 October 2020 / Accepted: 23 October 2020 / Published online: 31 October 2020 \\ (C) Neuroscientia 2020
}

\begin{abstract}
Given that the lockdown measures taken so as to diminish the dissemination of the COVID-19 pandemic are likely to create major mental disorders such as obsessive-compulsive disorder (OCD), the present study was conducted aiming to evaluate the prevalence of OCD and cognitive errors among young people during the outbreak of coronavirus disease 2019. The present study is a descriptive, cross-sectional study in which a total of 150 young people aged 13 to 19 were randomly included. Maudsley Obsessive-Compulsive Inventory Questionnaire (MOCI) and Cognitive Error Questionnaire (CEQ) were completed, and then their results were analyzed by SPSS 22 software. The results revealed that $67.3 \%$ of the subjects who were evaluated may have demonstrated OCD symptomatology. The prevalence of obsessive-compulsive disorder symptoms in women was slightly higher than in men (72.1 compared to $60.3 \%)$. Likewise, the test results indicated that there was a significant difference between obsessive-compulsive disorder symptom in men and women $(p=.001)$. There was also a significant difference between the two groups with OCD symptoms and without OCD symptoms in all the components of cognitive errors $(p=.001)$. To the authors' best of knowledge, this is the first study that has evaluated OCD symptoms at the time of COVID-19 prevalence. The findings suggest the need to enhance the prevention of relapse during social restrictions as well as to develop other strategies including online consultation and digital psychiatric management.
\end{abstract}

Keywords Cognitive errors · Obsessive-compulsive disorders · Young people $\cdot$ Coronavirus disease 2019

\section{Introduction}

Initially originated in Wuhan, coronavirus disease 2019 (COVID-19) has diffused throughout China since December 2019, posing serious threats to human health (Lai et al. 2020; Vakili et al. 2020). On January 30th, WHO declared that the novel coronavirus pneumonia epidemic (NCP, subsequently dubbed COVID-19) is a Public Health Emergency of International Concern ( $\mathrm{Wu}$ and McGoogan 2020).

\section{Sheida Jamalnia}

Shjamalnia1988@gmail.com

1 Child and Adolescent Psychology, Science and Arts University, Yazd, Iran

2 Department of Midwifery, Firoozabad Branch, Islamic Azad University, Firuzabad, Iran

3 Department of Psychology, Science and Arts University, Yazd, Iran

4 Medical Journalism Department, Shiraz University of Medical Sciences, Shiraz, Iran
According to the statistics, 11,206,261 confirmed and 529,403 death cases have been reported as of July 7 . As the global health agencies have been striving to come up with efficient treatment, the World Health Organization (WHO) and the Center for Disease Control and Prevention (CDC) suggest use of strategies such as social distancing, as well as hand-respiratory hygiene to the extent that even every mass media source is accentuating the utmost significance of hygienic measures, washing, and prevention of contamination (Lin et al. 2020). Therefore, given that the most prevalent manifestations of OCD are panic of contamination and excessive hand washing habit, coupled with considerably increasing emphasis on washing hands laid by health advisories, the signs of OCD are most likely to exacerbate (Alzyood et al. 2020). Of all the psychosocial implications caused by the COVID-19 pandemic including panic, health anxiety, mass hysteria, and lockdown loneliness, obsessive-compulsive symptoms have inadequately been taken into consideration (Fontenelle and Miguel 2020). 
Obsessive-compulsive disorder is, according to the DSM5 , repetitive and persistent thoughts, impulses or images that are perceived as distracting, unwelcome at any stage during the disruption, and that trigger marked anxiety or depression in most people. The individual attempts to ignore or suppress such thoughts, urges, or images, or to neutralize them with a certain thought or action (i.e., by performing a compulsion). One of the most common psychological disorders with two characteristics is obsessive-compulsive disorder: disturbing, uncontrollable, and repeated thoughts that cause anxiety in the individual (obsessive-compulsive disorder) and second, repetitive behaviors aimed at reducing this anxiety (practical obsession) (Abuse and Administration 2016).

According to multiple reports, OCD has an effect of 1 to $3 \%$ on children and adolescents and is associated with significant disabilities and reduced quality of life (Fontenelle et al. 2006; Ruscio et al. 2010). The illness has also been shown to be associated with high levels of comorbidity and significant psychosocial dysfunction in young people including low levels of concentration at school and difficulty completing homework and doing household routines, as well as impaired social functioning. If OCD-diagnosed young people do not gain sufficient treatment, their symptoms are highly likely to persist to the extent of experiencing further psychopathology in adulthood (Fineberg et al. 2020). Individuals who have OCD and are afraid of being contaminated by the virus may spend long hours worrying about the likelihood of contracting the infectious disease and preventing other potential contaminants, e.g., not touching certain surfaces or decreasing social interactions (Remmerswaal et al. 2019). The significance of preserving hand hygiene in suppressing the virus makes patients rationalize their behaviors which usually have a very ritualistic feature, facilitating their diagnosis by a mental health professional (Subramaniam et al. 2020).

Many studies have been performed over the past few decades to determine the psychological factors involved in the onset and development of obsessive-compulsive disorder (Jaisoorya et al. 2017). Each one of these studies, in some way, has considered one of the elements involved in these disorders. One of these components is cognitive errors which affect people's decision-making and judgment of situations. Instances of cognitive errors in individuals with practical obsessive-compulsive disorder involve overestimation of risk and high accountability (Meygouni 2001). Researches performed in the realm of cognitive errors display similar results; as an instance, it is revealed that cognitive errors worsen the severity of and predict obsessive-compulsive disorder (Salek Ebrahimi et al. 2019; Mousavi et al. 2017). Furthermore, concern with such thoughts, particularly high accountability thoughts, intensifies the symptoms of anxiety in obsessivecompulsive disorder (Salek Ebrahimi et al. 2019). A review conducted on obtrusive thoughts, obsessions, and stimulators in obsessive-compulsive disorder found that cognitive errors in anxiety disorders principally emanate from risk-taking and disaster-related behavior (Julien et al. 2007).

Accordingly, in the current crisis, it is considered that collecting high-quality documentation of the mental health needs of individuals is feasible and cost-effective. The number of individuals developing OCD and fearing COVID-19 infection might even increase in the next few months or years. As Iran's young people have been exposed to a tenacious source of anxiety and worry, the present research was performed aiming to indicate the psychological manifestations of their distress. To this end, the prevalence of obsessive-compulsive disorder and cognitive errors that contributed to its symptoms in young people were detected.

\section{Methodology}

The present study was of a descriptive, cross-sectional nature in which the statistical population was comprised of all high school and pre-university students. Given a 95\% confidence coefficient and the error rate of $5 \%$ based on the Cochran's formula, the sample size was a total of 150 people determined according to the number of the population under examination. The sampling procedure was performed randomly. The criteria for inclusion into the study were a minimum age of 13 and a maximum of 19 years, reading and writing literacy, and consent to participate in the study.

The criteria for exclusion were any history of personality disorders, mental retardation, bipolar disorder, psychosis, and substance abuse. All subjects filled in the Maudsley Obsessive-Compulsive Inventory Questionnaire (MOCI) and Cognitive Error Test (CET).

\section{Maudsley Obsessive Compulsive Inventory Questionnaire}

Maudsley Obsessive-Compulsive Inventory Questionnaire (MOCI), designed to measure obsessive-compulsive disorder, comprises 30 items that are answered in the form of a yes or no. MOCI is used as a screening tool for obsessivecompulsive symptoms in psychiatric patients and nonclinical populations. The questionnaire was invented in such a way that a completely obsessive person would respond to exactly half of the questions in the affirmative and the other half in the negative. Hodgson and Rachman (1977) managed to obtain four subgroups through factor analysis: two main types of complaint, checking and washing compulsions, and two minor ones, slowness and doubting, were established. Both in clinical trials and in the public population, this questionnaire was used. Total Maudsley score ranges from zero to 30. The MOCI has not been reported to be internally consistent nor factorially distinct, and coverage of OCD symptoms other than washing and checking (e.g., hoarding, convert 
rituals) is limited. Because of its dichotomous response structure and many things relating to past and permanent incidents, the MOCI is also not appropriate for assessing improvements in care (Taylor et al. 2004). Hodgson (1977 and 1980) assessed the convergence validity and reliability of the reexamination of this questionnaire as satisfactory and estimated at about 0.89 . Also, Cronbach's alpha coefficient reported for the total score as 0.87 . The content validity of this test has been reviewed and confirmed in Iran by Ghasemzadeh (2001). Aliloo (2006) has estimated its reliability on a group composed of 25 individuals as 0.82 .

\section{Cognitive Errors Questionnaire}

This Cognitive Errors Questionnaire (also called CET) was created by Meyghoni (2001) and standardized on 80 people from the military garrisons of Tehran. The CET is comprised of 50 10-subscale sentences: all-not thinking, mental filtering, overgeneralization, jumping to conclusion, labeling, neglecting positive points, catastrophizing, should statement, emotional reasoning, and personalization. Five appropriate items are developed for each one of these 10 cognitive errors. Told to mentally imagine and place themselves in the imaginarily established environment, subjects are asked to only select one item out of five modes for each topic. The answers will be scored using a Likert scale with a score of $4=$ completely agree, $3=$ agree, $2=$ no idea, $1=$ disagree, and $0=$ completely disagree. The high scores in the completely agree and agree items are indicative of cognitive errors compared to the test's norm. To determine the reliability coefficient of this instrument, Meyghoni (2001) randomly chose 20 subjects out of all the participants in the initial standardization phase for each category. He then, 2 weeks after the first test, tested the same group again using the same questionnaire. The average overall correlation coefficient between the first- and second-time responses for women was 0.61 and for men was 0.67 , and the mean correlation coefficient of total scores was 0.64. The collected data were then analyzed using SPSS 22.

\section{Results}

Of the 150 participants, $64.7 \%$ were women. The average age of women was 16.37 and that of men was 16.97 . The results revealed that $67.3 \%$ of the subjects may have demonstrated OCD symptomatology. The prevalence of obsessive-compulsive disorder symptoms in women was slightly higher than in men (72.1\% compared to $60.3 \%$ ). The test results displayed that there was a significant difference between the rate of obsession in men and women $(p=.001)$ (Table 1). Descriptive findings proved that the highest prevalence of obsessive-compulsive disorder symptom belonged to washing compulsion. All the groups under examination differed in terms of all the components of cognitive
Table 1 Descriptive indicators of obsessive-compulsive disorder and its components and the frequency distribution of OCD symptoms by gender $(n=150)$

\begin{tabular}{lllllll}
\hline Scale & Subscale & Mean & SD & Female & Male & $p$ value \\
\hline OCD & Checking & 4.8 & 1.4 & $5.1(1.2)$ & $4.1(1.4)$ & \\
& Washing & 6.3 & 1.6 & $6.7(1.7)$ & $5.8(1.3)$ & \\
& Strictness & 4.5 & 1.3 & $5.1(1.2)$ & $3.6(.7)$ & \\
& & & & & & .001 \\
& Doubting & 4.6 & 1.1 & $5.1(.9)$ & $3.7(.7)$ & \\
& & & & & & .001 \\
Total score & & 16.42 & 4.2 & $18.1(4)$. & $13.3(2.6)$ & \\
& & & & & & .001 \\
\hline
\end{tabular}

errors $(p=.001)$. In all the components where there was a significant difference between the means, the mean score for the OCD group was higher than those of the group without symptoms of practical obsessive-compulsive disorder. Also, in OCD subjects, the highest average cognitive error belonged to "Personalization" and the lowest to "Sensory argument" (Table 2).

\section{Discussion}

There is a significant difference between the groups in terms of all the components of cognitive errors. In all the components wherein, there was a significant difference between the means; the mean score of the OCD group was higher than that of non-OCD group. Also, in OCD subjects, the highest average cognitive error was "Personalization" and the lowest was "Sensory argument" (Table 2). The values for OCD subjects' mean and median age in the population were 16.67 and 16 years, respectively. Moreover, the time at which OCD begins, as reported by some studies, ranges from 15 to18 years of age.

An examination of the results of this study revealed that 67.3 subjects experienced OCD. Likewise, the study conducted by Khosravi et al. aiming to evaluate the prevalence of practical obsessive-compulsive disorder in Jahrom city indicated that OCD was observed in $43 \%$ of the subjects under examination. It should be noted that this value belongs to nonCOVID-19 period (Khosravi and Naseri 2017).

A total of $5.8 \%$ and $28.2 \%$, respectively, were the prevalence values recorded by the Netherlands Mental Health Survey and Incidence Research and the National Comorbidity Survey in the USA. This study revealed that the most common OCD sign was washing compulsion $(61 \%)$. In the USA, however, it was shown by Ruscio et al. that the most common manifestation was checking compulsion (15.4\%) (Ruscio et al. 2010). 
Table 2 The mean and standard deviation of cognitive error components in the OCD group and non-OCD group

\begin{tabular}{|c|c|c|c|}
\hline Cognitive error & $\begin{array}{l}\text { OCD group }(n=101) \\
\text { Mean }( \pm \mathrm{SD})\end{array}$ & $\begin{array}{l}\text { Non-OCD group }(N=49) \\
\text { Mean }( \pm \mathrm{SD})\end{array}$ & $p$ value \\
\hline All-or-nothing thinking & $13.3(1.6)$ & $9.3(1.0)$ & \\
\hline Mental filter & $12.6(1.2)$ & $9.8(.8)$ & \\
\hline Extreme generalization & $11.8(.7)$ & $10.4(1.3)$ & \\
\hline Hasty conclusions & $11.9(1.0)$ & $10.3(1.3)$ & \\
\hline Labeling others & $12.0(1.0)$ & $10.3(1.4)$ & \\
\hline Ignoring positive affairs & $11.4(1.1)$ & $9.5(1.4)$ & \\
\hline Catastrophizing & $11.3(1.3)$ & $9.4(1.2)$ & \\
\hline Dos & $12.6(1.5)$ & $10.4(1.5)$ & \\
\hline Sensory argument & $10.8(1.7)$ & $8.7(1.1)$ & \\
\hline Personalization & $13.9(1.3)$ & $10.41(1.5)$ & \\
\hline
\end{tabular}

The prevalence of eczema in young people as reported by Jaisoorya et al. was 3.3\%. Additionally (Jaisoorya et al. 2017), the study by Politis et al. (Politis et al. 2017) performed to assess the prevalence of OCD in young people showed an estimated prevalence of $1.39 \%$. These results, however, belong to non-COVID-19 times.

In several studies conducted in Iran, the prevalence of OCD in young people and adults has been reported to be more than $20 \%$, a rate higher than that of other countries; it was reported in the period prior to the outbreak of COVID-19 in Iran (Khosravi and Naseri 2017; Sarokhani et al. 2016). Given the potentially rising spread of this disease all over the world, the rate is expected to increase (Sarokhani et al. 2016).

The current COVID-19 pandemic is highly likely to affect young people and adults who are most susceptible to OCD development (Davide et al. 2020). When they discover that their repetitive, stereotypical, and long-term hand washing procedure, which is strongly recommended during the crisis, alleviates their anxiety, even those without any pre-existing problems unintentionally develop compulsive hand washing. It is mostly external events, related to current concerns, which give rise to obsessions (Wahl et al. 2020). During this outbreak, the number of relapsing OCD subjects in Iran is increasing, although this report comes from personal and anecdotal data gathered by most clinicians (Sarokhani et al. 2016).

Not only are not pandemics just biological or medical phenomena, but they have enormous psychosocial implications and have great influences on society at large. Since the WHO announced COVID-19 as a pandemic, accentuating washing and disinfection, the reports of OCD peoples' visits have been increasing as well (Alzyood et al. 2020).

Obsessions with developing contamination and compulsive hand washing are of the most widespread of many manifestation domains. It has long been shown that people with pre-existing psychiatric illnesses are more likely to start relapses, as well as failure during pandemics to take drugs, stress, stigma, inadequate self-care, and suicide (Davide et al. 2020).

Another point that deserves special attention is the evaluation of excessively high responsibility beliefs as a mediator between signs of contamination and those signifying intensified status as they have been shown to be cognitive/ maintenance vulnerability factors that are specific to OCD (Pozza et al. 2017). We may conclude that during the lockdown at home, the conditions for OCD-diagnosed individuals with extremely excessive obligation beliefs and raised contamination obsessions eventually deteriorate (Lin et al. 2020). However, the conditions for those with only signs of contamination who do not fear of spreading their contamination to others will not experience such a worsening trend.

Subjects with anxiety disorders and OCD experience cognitive errors in their thought process (Davide et al. 2020). Likewise, the central theme of cognitive error highlights obtrusive thinking, obsessions, and stimulants in OCD. Cognitive errors are the chief characteristics of a wide variety of anxiety disorders, such as OCD. All cognitive theories have accentuated the role of an important factor in creating and maintaining anxiety disorders, which is the existence of cognitive errors (Mousavi et al. 2017). 
The findings of the present study showed that there is a significant difference between the mean of all cognitive error components in the two OCD and non-OCD groups. These findings are consistent with those of Mousavi's study wherein it was shown that cognitive errors increased the severity of and predicted OCD and that the presence of these thoughts, especially those with inflated levels of responsibility, exacerbated OCD anxiety symptoms (Mousavi et al. 2017). Cognitive errors, along with a lack of social skills, play a key role in the onset and stabilization of this disorder (Meygouni 2001).

We fell short of exactly showing if such heightened symptoms happened only to subjects with OCD due to the lack of a non-clinical control group without OCD or even another psychiatric group. In addition, the inevitable shortage of a control group composed of subjects, who were not exposed to the lockdown, prevented us from making a distinction between the effects of the lockdown and those of other potential sources of bias including lack of psychiatric visits during the lockdown, the insufficiency of the living physical space, or financial worries generated by the COVID-19 pandemic.

The OCD worsening has been profoundly reported in the former outbreaks like severe acute respiratory syndrome (SARS), Middle East respiratory syndrome (MERS), and influenza, even within 6 to 12 months after the outbreak ended (Chong et al. 2004). Whenever the countermeasures against an infection require "repetitive behaviors," the likelihood of developing obsessional disorders is increased, which might not be obviously discernable at the active phase of the outbreak due to under-detection, disruption of medical services, and alternate public health priorities.

The priority of public health officials and mass media during pandemics is on the outbreak's biological and physical implications rather than mental health concerns. Further demands have been made for measures to increase public support for mental health (Tang et al. 2018). According to the Pew Research Centre (2015), at least $92 \%$ of teenagers are active on social media. Extensive coverage of the pandemic by the mass media often affects the public's physical and psychological reaction to the danger of infectious diseases, which may intensify their anxiety even though they are advised to take precautionary and protective measures to protect themselves from the virus (Rodin et al. 2019). To minimize the impact of media on mental health, the government and health authorities must provide timely and reliable evidencebased information about the pandemic to the public through traditional and new media.

As various mental health matters and needs are less likely to be among the major priorities of the public health agenda during the active stage of a pandemic, it will result in worsening of mental health disorders symptoms and late diagnoses. Many OCD subjects might have become aware of the clues to their disorders, but are reluctant to ask for assistance either due to stigma or unawareness of what "excess" behavior means in terms of washing and bathing (Davide et al. 2020). Nevertheless, as an increased number of countries are being affected during the ongoing lockdown days, it has turned into an utmost necessity to manage and address mental disorders to maintain the quality of lives.

Considering the increased demand to carefully wash hands in the minimum period of time, it should be recommended, for example, to restrict access to news, or view them only through trustworthy sources, spend time daily on pleasant or physical activities which contribute to distracting intrusive thoughts and lowering the level of anxiety. Furthermore, the lockdown and the limited access to mental health centers have possibly dissuaded subjects from asking for assistance and have caused delays in the interventions.

The necessary measures that can be taken to assist the OCD subjects in need of help include psychoeducation of the OCD subjects and families, debunking misinformation about the pandemic, facilitating video-consults and online psychotherapy, and use of psychiatric social work support to follow up on severe and treatment-resistant OCD subjects so as to ensure their drug compliance. Consequent anxiety might result in the obsessive use of medications like hydroxychloroquine (HCQ), which has been recently presented in guidelines for COVID-19.

\section{Conclusion}

The primary health-care workers are strongly recommended, whatever and wherever their responsibilities lie, to have training in identifying OCD complaints and the necessary referrals. It is inevitable that the pandemic will eventually subside; however, the increased incidence and intensity of mental health disorders, especially OCD, will be the dire consequences for many months to come.

This study, like other ones, had limitations. One of the major limitations of this study was the large number of questions in the questionnaires. In this regard, we tried to control the effect of fatigue by randomly presenting the questionnaires.

\section{Compliance with Ethical Standards}

Conflict of Interest The authors declare that they have no conflict of interest.

\section{References}

Lai, C-C, Shih, T-P, Ko, W-C, Tang, H-J, Hsueh, P-R. (2020) Severe acute respiratory syndrome coronavirus 2 (SARS-CoV-2) and corona virus disease-2019 (COVID-19): the epidemic and the challenges. International journal of antimicrobial agents.:105924. 
Vakili, S., Savardashtaki, A., Jamalnia, S., Tabrizi, R., Nematollahi, M.H., Jafarinia, M., et al.. (2020) Laboratory findings of COVID19 infection are conflicting in different age groups and pregnant women: a literature review. Archives of Medical Research.

Wu, Z., McGoogan, J.M.. (2020) Characteristics of and important lessons from the coronavirus disease 2019 (COVID-19) outbreak in China: aummary of a report of 72314 cases from the Chinese Center for Disease Control and Prevention. Jama.

Lin, Y-H, Liu, C-H, Chiu, Y-C. (2020) Google searches for the keywords of "wash hands" predict the speed of national spread of COVID-19 outbreak among 21 countries. Brain, Behavior, and Immunity.

Alzyood, M., Jackson, D., Aveyard, H., Brooke J. (2020) COVID-19 reinforces the importance of hand washing. Journal of Clinical Nursing.

Fontenelle, L.F., Miguel, E.C.. (2020) The impact of COVID-19 in the diagnosis and treatment of obsessive-compulsive disorder. Depression and Anxiety.

Abuse, S., Administration, M.H.S.. (2016) Impact of the DSM-IV to DSM-5 changes on the National Survey on Drug Use and Health.

Fontenelle, L. F., Mendlowicz, M. V., \& Versiani, M. (2006). The descriptive epidemiology of obsessive-compulsive disorder. Progress in Neuro-Psychopharmacology and Biological Psychiatry., 30(3), 327-337.

Ruscio, A. M., Stein, D. J., Chiu, W. T., \& Kessler, R. C. (2010). The epidemiology of obsessive-compulsive disorder in the National Comorbidity Survey Replication. Molecular Psychiatry, 15(1), $53-63$.

Fineberg, N.A., Van Ameringen M., Drummond, L., Hollander, E., Stein, D., Geller, D., et al.. (2020) How to manage obsessive-compulsive disorder (OCD) under COVID-19: a clinician's guide from the International College of Obsessive Compulsive Spectrum Disorders (ICOCS) and the Obsessive-Compulsive Research Network (OCRN) of the European College of Neuropsychopharmacology. Comprehensive Psychiatry.

Remmerswaal, K. C., Batelaan, N. M., Hoogendoorn, A. W., van der Wee, N. J., van Oppen, P., \& van Balkom, A. J. (2019). Four-year course of quality of life and obsessive-compulsive disorder. Social Psychiatry and Psychiatric Epidemiology., 1-12.

Subramaniam, M., Abdin, E., Vaingankar, J., Shafie, S., Chang, S., Seow, E., et al. (2020). Obsessive-compulsive disorder in Singapore: prevalence, comorbidity, quality of life and social support. Annals of the Academy of Medicine, Singapore, 49(1), 15.

Jaisoorya, T., Reddy, Y. J., Nair, B. S., Rani, A., Menon, P. G., Revamma, M., et al. (2017). Prevalence and correlates of obsessive-compulsive disorder and subthreshold obsessivecompulsive disorder among college students in Kerala, India. Indian Journal of Psychiatry, 59(1), 56.

Meygouni, D. (2001) The relationship between personality catagories and cognitive errors: MSc. thesis]. Tehran: Iran University of Medical Sciences.

Salek Ebrahimi, L., Mousavi, S. E., Gharraee, B., Mohammadi Bytamar, J., \& Saberi, I. M. (2019). Cognitive errors and psychological resilience in patients with social anxiety and obsessive-compulsive disorder: a cross-sectional study. Hospital Practices and Research., 4(1), 25-30.

Mousavi, E., Gharraee, B., Ramazani Farani, A., \& Taremian, F. (2017). Comparison of cognitive errors and rumination in obsessivecompulsive and social phobia disorders. Iranian Journal of Psychiatry and Clinical Psychology., 23(1), 10-21.

Julien, D., O'Connor, K. P., \& Aardema, F. (2007). Intrusive thoughts, obsessions, and appraisals in obsessive-compulsive disorder: a critical review. Clinical Psychology Review., 27(3), 366-383.

Taylor, S., Thordarson, D., Sã, I., Antony, M., Barlow, D.. (2004) Handbook of assessment and treatment planning for psychological disorders.

Khosravi, S., \& Naseri, A. (2017). Obsessive-compulsive prevalence investigation in Jahrom city. Journal of Jahrom University of Medical Sciences., 15(3), 1-8.

Politis, S., Magklara, K., Petrikis, P., Michalis, G., Simos, G., \& Skapinakis, P. (2017). Epidemiology and comorbidity of obsessive-compulsive disorder in late adolescence: a crosssectional study in senior high schools in Greece. International Journal of psychiatry in clinical practice., 21(3), 188-194.

Sarokhani, D., Sarokhani, M., Sayehmiri, K., \& Azodi, M. Z. (2016). The evaluation of obsessive-compulsive disorder prevalence in Iran: a meta-analysis and systematic review. International Journal of Pharmacy \& Technology., 8(4), 21482-21492.

Davide, P., Andrea, P., Martina, O., Andrea, E., Davide, D., Mario, A.. (2020) The impact of the COVID-19 pandemic on patients with OCD: effects of contamination symptoms and remission state before the quarantine in a preliminary naturalistic study. Psychiatry Research:113213.

Wahl, K., Salkovskis, P., Allegro, F., Miché, M., Kordon, A., Lieb, R., et al. (2020) Cognitive motivations for the initiation of ritualistic hand washing in obsessive-compulsive disorder. Cognitive Therapy and Research.:1-11.

Pozza, A., Barcaccia, B., \& Dèttore, D. (2017). The Obsessive Compulsive Inventory-Child Version (OCI-CV): further evidence on confirmatory factor analytic structure, incremental and criterion validity in Italian community children and adolescents. Archives of psychiatric nursing., 31(3), 291-295.

Chong, M.-Y., Wang, W.-C., Hsieh, W.-C., Lee, C.-Y., Chiu, N.-M., Yeh, W.-C., et al. (2004). Psychological impact of severe acute respiratory syndrome on health workers in a tertiary hospital. The British Journal of Psychiatry., 185(2), 127-133.

Tang, L., Bie, B., Park, S.-E., \& Zhi, D. (2018). Social media and outbreaks of emerging infectious diseases: a systematic review of literature. American journal of infection control., 46(9), 962-972.

Rodin, P., Ghersetti, M., \& Odén, T. (2019). Disentangling rhetorical subarenas of public health crisis communication: a study of the 2014-2015 Ebola outbreak in the news media and social media in Sweden. Journal of Contingencies and Crisis Management., 27(3), 237-246.

Publisher's Note Springer Nature remains neutral with regard to jurisdictional claims in published maps and institutional affiliations. 